\title{
REFLEXIONES SOBRE LA FUNCIÓN DOCENTE DEL PROFESOR UNIVERSITARIO
}

\section{REFLECTIONS ON THE ROLE OF PROFESSOR UNIVERSITY \\ TEACHER}

\section{AUTORES}

Michel Heykoop Fung-A-You: Prof. Titular y Director del Departamento de Biología Vegetal. Universidad de Alcalá.

michel.heykoop@uah.es

\section{CURRÍCULUM VITAE}

Profesor Titular de Universidad del Departamento de Biología Vegetal y Vicerrector de Postgrado y Campus de Guadalajara de la Universidad de Alcalá de Henares (España).

\section{RESUMEN}

Con un (ante)proyecto de Ley de Universidades a las puertas del parlamento e inmersos en un intenso debate sobre el estado y los males que aquejan a la Universidad española, parece conveniente reflexionar, siquiera sucintamente, sobre una de las funciones primordiales de esta Institución como es la docencia.

\section{PALABRAS CLAVE}

Reflexiones - Profesor - Función docente 


\begin{abstract}
With a (pre) Universities Bill at the gates of parliament and immersed in an intense debate on the state and the evils afflicting the Spanish university, it seems appropriate to reflect, even briefly, on one of the primary functions of this institution as teaching.
\end{abstract}

\title{
KEY WORDS
}

Reflections - Teacher - Teaching Function

Con un (ante)proyecto de Ley de Universidades a las puertas del parlamento e inmersos en un intenso debate sobre el estado y los males que aquejan a la Universidad española, parece conveniente reflexionar, siquiera sucintamente, sobre una de las funciones primordiales de esta Institución como es la docencia.

En general el debate que se está produciendo, y su traducción en el citado anteproyecto de ley, se ha centrado excesivamente sobre algunas cuestiones como son, por ejemplo, la endogamia en la selección del profesorado o la reforma de los órganos de gobierno dejando de lado otros problemas mucho más graves. Así, por ejemplo, se echa especialmente de menos un plan de financiación adecuado para una Universidad que, en el contexto europeo, se encuentra prácticamente a la cola del ránking en cuanto a porcentaje del PIB dedicado a la misma se refiere. Esto resulta 
paradójico ya que ¿cómo se pueden solucionar de verdad los problemas que nos aquejan si el coste de la reforma parece ser cero?

Por otro lado, como ya dijera Ortega y Gasset (1930) "la raíz de la reforma universitaria está en acertar plenamente con su misión", y en este sentido todos tenemos que reflexionar sobre la misión que debiéramos desempeñar en el seno de la Universidad. Por ello, en calidad de profesor voy a tratar de analizar algunos de los problemas recurrentes que salen a relucir, una y otra vez, en círculos de debate más reducidos que los mediáticos y que son absolutamente cruciales en relación con lo que se supone es nuestra misión como profesores universitarios.

En primer lugar, a diferencia de lo que cree el gran público, nuestra función es doble. Me estoy refiriendo al hecho de que "a pesar" de ser profesores nuestra misión no es exclusivamente docente, sino que conlleva también una actividad investigadora. Ese es un hecho fundamental en el que no se ha profundizado lo suficientemente en este país, y que, desde luego, la sociedad en general no ha sabido comprender. Desarrollamos un servicio a la sociedad que consiste en formar futuros científicos, profesionales, y, en general, personas, además de producir y crear conocimiento por lo que en teoría cumplimos una función básica en el sistema. ¿Por qué entonces este sentimiento de frustración e incluso de fracaso en el profesorado? ¿Por qué este claro desprestigio de la figura del docente en general y del profesor universitario en particular? Estas son dos preguntas claves en el debate y que hoy por hoy no han tenido una respuesta satisfactoria.

Muchos sectores de la sociedad nos tachan de ser unos trabajadores privilegiados, que gozan de muchas vacaciones, que trabajan poco, con una seguridad laboral de la que carece la mayoría, y que, en consecuencia, no tienen derecho a quejarse por nada a pesar de cobrar unos sueldos "miserables". Preguntas como: ¿a qué te dedicas a partir del 1 de junio si ya han terminado las clases? o ¿cuántas horas de clase tienes 
que impartir al día?, etc., son tan frecuentes que uno se desespera dando explicaciones una y otra vez al lego en la materia. Por otra parte, éste nunca acaba de creerse del todo nuestras explicaciones, por lo que, al final, acabas por ignorar o eludir ese tipo de discusión. He aquí, pues, uno de los primeros aspectos que debería ser sometido a un serio debate para tratar de configurar con más precisión nuestra función en la Universidad. ¿Cuánto de Profesor y cuánto de Investigador debemos ser? ¿Es posible ser Profesor e Investigador, al mismo tiempo y con un mínimo de rigor? En esta doble función ¿cuál de ellas debe primar, si es que alguna debe hacerlo?

Actualmente se habla mucho de la necesidad de incrementar la calidad de las Universidades haciendo especial hincapié en la importancia y papel clave de la investigación como indicador inequívoco de la misma; sin embargo, en este discurso se deja bastante de lado el aspecto de la calidad docente... A título de ejemplo, recientemente un grupo de Catedráticos encabezados por el Prof. Tortella (2001) propone un modelo universitario en el que la responsabilidad de la toma de decisiones recaiga exclusivamente en aquellos profesores de más calidad, y para establecer dicha calidad sugiere, ¡nada menos!, que se clasifique a todo el personal docente en diferentes niveles basados en el número de sexenios (tramos de investigación) obtenidos por los mismos. Es decir la calidad del profesorado se establecería exclusivamente en función de su excelencia investigadora... Afortunadamente existen otros profesores, entre los cuales me incluyo, que no sólo consideran totalmente necesario la valoración rigurosa de la labor investigadora, sino que valoran en igual medida la excelencia docente la cual, ¡obviamente!, no se establece en función de los sexenios. En este sentido, son muy interesantes los puntos de vista de profesores como por ejemplo Yela (2000) y Michavila \& Calvo (2000) o los de García Garrido (2001) que al analizar la carta abierta de Tortella manifiesta: 
"(...) la universidad es una institución con finalidad docente, una institución de aprendizaje. Tan claves resultan ser la calidad de la investigación como la calidad de la docencia. Hay profesores que se refugian en la calidad de su investigación tratando de justificar así la falta de calidad de su docencia (falta de preparación de sus clases, atención cicatera al alumnado, etc.)".

En relación con todo lo anterior, no deja de ser sorprendente que nuestros cargos de funcionarios reciban el nombre de "profesores de Universidad" cuando lo que prima cada vez más es la labor investigadora. Lo primero que choca es que para acceder a los cuerpos docentes nos van a valorar, casi exclusivamente, nuestro currículum investigador. El número y calidad de nuestras publicaciones es prácticamente lo único que el tribunal va a tener en cuenta en la inmensa mayoría de las oposiciones y concursos. No importa si un profesor tiene unos méritos docentes excepcionales, no se tienen en cuenta. Además, ¿de qué manera puede un tribunal o una comisión valorar esos méritos docentes? Como mucho podrá verificar los años de docencia y el nombre de las asignaturas impartidas por el candidato. Claro, a los "brillantes pensadores" del Ministerio de Educación se les ocurrió que esta deficiencia debía de ser paliada de alguna manera y se estableció un sistema de complementos por méritos docentes, los cuales serían adjudicados mediante una supuesta evaluación consistente en unas encuestas a las que, todos los cursos académicos, se les somete a los estudiantes. ¿Sirve este método? Hay opiniones para todos los gustos. Casi siempre sesgadas y dependientes del resultado personal del profesor en cada caso.

Desde mi punto de vista creo sinceramente que, con todas las deficiencias y errores que pudiera tener este sistema de encuestas (deficiencias subsanables por otra parte), sí que sirven y que debieran ser tenidas en cuenta. El valor de dichas encuestas, por supuesto, no es absoluto sino relativo, pero claramente -sobre todo si se analizan considerando un periodo lo suficientemente largo, no de un solo curso académicoindican unas tendencias y pueden arrojar mucha luz sobre la labor desempeñada por 
cada profesor. ¿Por qué entonces no se tienen en cuenta a la hora de adjudicar el complemento por méritos docentes (quinquenio)? Algunos argumentan, no sin cierta razón, que ese complemento constituye una subida de sueldo encubierta. Lo correcto sería solucionar de una vez por todas el problema de retribuir dignamente al profesorado, y a partir de allí establecer una evaluación docente rigurosa que, naturalmente, no puede ser llevada a cabo por nosotros mismos, sino que debería ser externa al igual que la evaluación de nuestra labor investigadora.

Deben establecerse diferencias a la hora de la adjudicación de los complementos por docencia, del mismo modo a cómo se establecen en las evaluaciones de la actividad investigadora. Lo importante está en acertar en el método evaluador, y me refiero a la evaluación del profesor, no a la de la evaluación institucional que esa sí se está llevando a cabo en algunas licenciaturas.

Veamos algunas opiniones al respecto. Michavila \& Calvo (2000) indican que:

"A diferencia del rigor y prestigio que ha adquirido la evaluación de la actividad investigadora de los profesores universitarios, la evaluación de su función docente se ha caracterizado, en general, por una exigencia mínima y en muchas universidades es un mero trámite automático, que cada cinco años permite obtener un pequeño incremento salarial. Se podría incorporar una segunda evaluación, adicional, de la docencia, selectiva, rigurosa y exigente, (...) Permitiría reducir el desequilibrio actual en la valoración y el interés que se presta en mucha mayor medida a las tareas investigadoras que a las docentes."

Yela (2000) sugiere:

"En mi opinión, debería tenderse a la creación de un organismo riguroso e independiente, al estilo de la ANEP, o que fuera parte de esta misma agencia, cuyos 
juicios sobre la calidad docente (...) fueran aplicables tanto a los profesores numerarios como a los no numerarios, y tuviera consecuencias sobre ambos colectivos $(\ldots)^{\prime \prime}$.

Esta misma problemática existe en países tan avanzados como los Estados Unidos de América donde científicos como Lenhoff (1996) ponen de manifiesto que para mejorar la docencia en las universidades americanas, y que ésta no se vea perjudicada en favor de la investigación, se debería obligar a todos los profesores que vayan a solicitar financiación para su investigación a la NSF (National Science Foundation) que, junto a sus solicitudes de proyectos, adjunten las evaluaciones realizadas por sus estudiantes de licenciatura y que ya son utilizadas como mérito y criterio de promoción por sus Departamentos y Decanos. Todas estas opiniones apuntan a la necesidad de que de alguna manera se evalúe rigurosamente la actividad docente del profesorado para evitar su progresiva degradación.

Ahora bien, aquellos que consideren que el único problema es el del incentivo cometen el error de caer en una visión demasiado simplista. La falta de incentivos, económicos se entiende, no es la causa única de los problemas de la docencia. También existe un problema vocacional como muy bien apunta José Luis Sampedro (1997) -catedrático, jubilado, de Estructura Económica y novelista-:

"no se debe ser profesor pensando en el beneficio económico o en otras satisfacciones. La remuneración es el hecho de dar clase y el poder relacionarse con los alumnos".

Efectivamente, la actividad docente constituye una de las actividades más enriquecedoras, desde el punto de vista personal, que se pueda imaginar, porque ¿qué puede ser más satisfactorio que estar colaborando en formar ciudadanos más cultos, educados y, por tanto, más libres? ¿Qué profesor, al menos el que es 
vocacional, no ha sentido alguna vez esa sensación de bienestar, indescriptible, esa sensación de éxito y triunfo después de una clase bien impartida?

Sea como fuere, y volvemos al principio, el problema básico es nuestra doble condición de profesor/investigador. En este sentido, hay dos visiones muy claras: por un lado, 1) aquellos que creen que no se puede ser profesor universitario sin realizar investigación científica; y, por otro 2) aquellos que creen que ambas actividades debieran estar claramente separadas. Sirva como ejemplo la opinión de Gerd Binnig (1989), premio Nobel de Física en 1986 y Profesor de la Universidad de Munich, que apunta lo siguiente:

"Creo que la actividad docente y la investigadora deberían aislarse más una de otra. Actualmente ambas actividades exigen tanto que una persona que intenta a conciencia llevar ambas a cabo sentirá por lo general que es superior a sus fuerzas."

¡Interesante punto de vista...! Y prosigue:

"Sin embargo, depende de cómo sea de drástica la separación. De hecho, creo que en el caso de una separación total, tanto de la investigación como de la enseñanza, faltaría algo. Sin investigación, a la enseñanza le falta actualidad, la sal y pimienta, la fascinación, todo sería más estilo colegio. Para hacer más comprensible lo que pienso, vaya un ejemplo: también la enseñanza puede resultar interesante. Si el lector acude al circo, su experiencia del mismo será distinta si consigue que sea un guía oficial o el director del circo (...) quien le guíe. Lo que resulta fascinante es la información directa. Por otro lado, a la investigación sin enseñanza le faltan los estudiantes, aparte de cuando se es profesor, se aprende mientras se enseña."

Esto último, claro, siempre que se sea lo suficientemente humilde y se esté dispuesto a recibir enseñanzas, actitud que no siempre se da entre los profesores de 
Universidad. ¿Cómo podríamos solucionar este problema central? He aquí la propuesta de Binnig:

"Para separar más la actividad investigadora de la actividad docente sin por ello separar investigación y enseñanza, cabría imaginar lo siguiente: hay profesores que se dedican a enseñar y otros a investigar. La frontera entre ambos ni siquiera tiene que estar bien definida, es decir, que sigue habiendo profesores que hacen ambas cosas. Pero ambos tipos de profesores se encuentran ahora reunidos en una organización (...) interactúan unos con otros. Cuando un profesor así lo desea, puede en calidad de docente contribuir a la enseñanza; pero no está obligado a hacerlo. De todos modos es cierto que hay quien le interesa más lo uno o lo otro. (...) Ahora, como equivalente, debería darse la posibilidad a un investigador para que pueda dedicarse principalmente o por completo a la enseñanza."

Mi opinión personal es que, ¡naturalmente!, un profesor universitario debe también desarrollar una actividad investigadora. La clave está en que tengamos siempre presente que ante todo somos docentes (sin alumnos no habría universidades) y que lo ideal sería que se valoraran en igualdad de condiciones ambas actividades, y cuando digo valorar me refiero a que, por ejemplo, se tengan en cuenta para los cómputos de la carga docente no sólo las horas de docencia impartidas sino todo lo relacionado con esa actividad: preparación de clases, tutorías (¡eso sí, tutorías serias!), participación en tercer ciclo, etc. ¿Cómo es posible, por ejemplo, que por ley tengamos que impartir 8 horas de docencia a la semana y dedicar además 6 horas a tutorías, y que luego la misma ley establezca que nuestra dedicación investigadora los famosos EJC (equivalentes a jornada completa)- sea de 32 horas? ¡Eso constituye una contradicción intrínseca que va contra la calidad docente! 
Por otro lado, deben establecerse los instrumentos adecuados para una evaluación justa de la actividad docente -la de la actividad investigadora, con todas sus "pegas" (véase el artículo de Gómez Caridad \& Bordons Gangas, 1996), está bien desarrollada- y que a la hora de presentarse a cualquier concurso de una plaza o promoción el resultado de la valoración de los candidatos derive de la valoración equilibrada de ambas actividades. Sólo así se podrán corregir las disfunciones del sistema resultantes de la falta de interés, generalizada, por la actividad docente.

Con todo, no sólo es suficiente para solucionar el problema que estamos analizando evaluar la actividad docente. Ésta también debiera de someterse a una intensa revisión en el marco de todo este debate de reforma universitaria.

La docencia, en nuestras universidades, adolece de toda una serie de problemas ancestrales y que tienen consecuencias muy negativas. Y no me estoy refiriendo ahora al problema que hemos analizado más arriba. Incluso en la labor de todos esos profesores que, sí, se toman en serio la actividad docente aparecen signos alarmantes que ponen de manifiesto la necesidad de una reforma exhaustiva. La cuestión central sobre la que, a mi juicio, debería girar este debate y que tendría que modificarse es la "pasividad" del método docente de nuestras universidades, y de la cual derivan otros muchos problemas.

Aquí, conviene señalarlo, también tienen su parte de culpa los estudiantes y, en última instancia, la Sociedad que concibe la Universidad exclusivamente como un lugar donde se expiden títulos para habilitar a profesionales. ¿A qué me refiero al hablar de "pasividad"?, pues sencillamente a que el método de enseñanza se reduce a la mera transmisión de conocimientos, que son asimilados de forma totalmente pasiva por los estudiantes, en detrimento de la estimulación de la creatividad en su sentido más amplio. Muchos profesores se limitan a dictar unos apuntes mientras que los estudiantes se obsesionan con copiar todo lo que se les dicta. Así, los 
profesores se convierten en "dictantes" y los estudiantes en "copiantes" perdiendo la condición de docentes y discentes. Veamos algunas opiniones al respecto: Paul Kurtz -vicepresidente de la Unión Humanista y Ética Internacional- indica en una entrevista publicada en El País (1995) que:

"La mejor garantía de la democracia es que todo el mundo esté capacitado para pensar por sí mismo. (...) Si yo estuviera en el gobierno español, pondría mucho dinero en la educación científica, no en el conocimiento en sí, sino en enseñar a pensar. La ciencia es una forma creativa de indagar, e incluye la duda y el escepticismo. Su enseñanza debe ser la prioridad número uno, porque no sobrevivirá ninguna economía si se retrasa respecto a la tecnología científica."

Por su parte, Emilio Lledó (2000) -filósofo- manifiesta respecto de la Universidad española:

"(...) la universidad española, atiborrada de planes de estudios encorsetados y ridículos, agobiada de exámenes (...), de apuntes, de profesores manualescos, obligados a dar programas completos de vaciedades infinitas que sañudamente se exigían en esos esterilizados exámenes. No pretendo, con esto, hacer una crítica fácil de una buena parte de nuestra universidad necesitada, como todo el sistema de enseñanza, de una verdadera reforma. Me sorprende, sin embargo, que se quiera plantear una cierta renovación convirtiéndola en una institución manoseada con criterios gerenciales o mercantiles. Un error gravísimo, fruto de un arbitrismo ignorante y confuso, muy alejado de la vida intelectual y de la cultura moral, que pasará por alto los verdaderos males, y que acabará hundiendo a la educación en el pantano de los dividendos y ganancias, en lugar de hacerla fructificar como semillero de libertad, de creatividad, de innovación y de sabiduría." 
Francisco Michavila -anterior secretario general del Consejo de Universidades y Catedrático de Matemática Aplicada- manifiesta en una entrevista, publicada en Menos 25 (1999), que:

"(...) los profesores (...) deben mejorar los sistemas de evaluación, se debe sustituir la enseñanza pasiva por otra activa y práctica...".

Volvamos ahora al libro de Gerd Binnig, comentado anteriormente. Según este autor la creatividad humana es un juego de alternancia entre síntesis y análisis, donde la síntesis sería el intento de buscar nuevas unidades de acción, mientras que el análisis consistiría en descubrir si el resultado realmente es una nueva unidad de acción y ver cuál es su importancia. En este sentido, Binnig opina que tanto en las escuelas como en las universidades no educan nuestra capacidad de síntesis ni de análisis; es decir, no nos familiarizan en absoluto con la creatividad. Al contrario, en la escuela y la universidad se nos dan los resultados que son unidades de acción establecidas. Según él:

"(...) tampoco se espera de nosotros que luego nos informemos sobre estos resultados. Sin duda alguna vez se produce una discusión de este tipo, pero muy rara vez. Y lo que realmente no sucede nunca es que nos inviten a "fantasear", a crear algo nuevo."

Es decir, resulta muy interesante dedicarse a otras actividades como, por ejemplo, componer música, escribir versos o pintar cuadros, ya que los mecanismos que conducen a la creatividad en el arte son exactamente los mismos que los que conducen a la creatividad en la ciencia. El "material" es diferente pero el "juego" que se entabla con él es el mismo. Es evidente que en nuestras universidades, y en nuestros métodos docentes, se pone el acento, casi exclusivamente, en la transmisión de conocimientos. No deja de ser sorprendente que en el sistema actual de oposición a Profesor de Universidad el primer ejercicio consista en una valoración y defensa del 
currículum vitae (casi exclusivamente de la parte investigadora) del candidato, seguida de la exposición de un programa docente, es decir, haciendo hincapié en los conocimientos que se piensan transmitir a los estudiantes. El segundo ejercicio consiste exclusivamente en la exposición de uno de los temas incluidos en el programa mencionado antes. No se aprecia ni valora la capacidad docente. El opositor va a lucirse exponiendo casi todo lo que sabe sobre el tema en cuestión. Como se ve exclusivamente méritos investigadores y transmisión de conocimientos. ¿Dónde quedan todas las cuestiones relacionadas con la metodología docente, la creatividad o el método científico? ¿Qué pasa con la filosofía de la ciencia?, otro aspecto que poco o nada se tiene en cuenta. $Y$, sin embargo, si lo que pretendemos (al menos en las carreras experimentales) es formar científicos, la filosofía de la ciencia no debería sernos ajena...

Vayamos, finalmente, al otro elemento fundamental de la docencia: los estudiantes. Como he dicho antes, el estudiante tiene su parte de culpa en la problemática de la docencia. El carácter pasivo de la docencia tiene su origen, en parte, en el método docente de los profesores, pero la actitud del estudiante es, por lo general, también absolutamente pasiva. A menudo, lo único que ambiciona éste es lograr obtener un título académico que le faculte para desempeñar una profesión, pero sin mostrar el más mínimo interés por el conocimiento o cultura en sí mismo o, lo que es peor, en llegar a ser una persona mejor, en el más amplio sentido de la palabra, que cuando entró en la Universidad. Es decir, los estudiantes están enfermos de "titulitis" y no parecen querer, en general, curarse de esta enfermedad.

Por supuesto, como ya he apuntado, mucha de la culpa es nuestra, de los profesores, ya que en nuestras manos está el tratar de estimularlos y atraerlos hacia la senda correcta. Eso sí, para ello es absolutamente necesario poner menos énfasis en la transmisión de conocimientos y potenciar la transmisión de unos "modos de actuar" frente a los problemas, en este caso de la ciencia. Debemos hacer comprender a los 
estudiantes que lo más importante, la lección primordial, es que deben estudiar para ser persona y no para ser algo. Ahí está el quid de la cuestión: una enseñanza que no sea pasiva sino interactiva, en la que participen ambas partes. De esa manera, y esa es la parte más remuneradora de todo el proceso, podremos lograr un cambio de actitud del estudiante frente a la vida, estimulando su curiosidad, su creatividad, su capacidad de plantear y resolver problemas, su capacidad -en definitiva- de pensar, lo cual unido por supuesto a los conocimientos específicos de la materia que impartimos le servirán de bagaje para su vida futura que comienza después de su paso por la Universidad.

\section{BIBLIOGRAFÍA}

BINNIG, G. (1989).- Desde la Nada. Sobre la creatividad de la naturaleza y del ser humano. Círculo de Lectores S.A. Madrid.

EL PAÍS (1995).- Científicos y filósofos abogan por la razón frente a lo religioso y lo paranormal. El País 23 de abril de 1995.

GARCÍA GARRIDO, J.L. (2001).- Una Universidad mejor. ABC, 12 de junio de 2001.

GÓMEZ CARIDAD, I. \& M. BORDONS GANGAS (1996).- Limitaciones en el uso de los indicadores bibliométricos para la evaluación científica. Política Científica 46:2126.

LENHOFF, H.M. (1996).- Letters. Promoting teaching. Science 273:721.

LLEDÓ, E. (2000).- Reflexiones sobre unos primeros recuerdos de Gadamer. El País, 25 de marzo de 2000. 
MENOS 25 (1999) Entrevista a Francisco Michavila, Secretario General del Consejo de Universidades. Menos 25, 10 de Abril de 1999.

MICHAVILA, F. \& B. CALVO (2000).- La Universidad Española hacia Europa. Fundación Alfonso Martín Escudero. Madrid.

ORTEGA Y GASSET, J. (1930).- Misión de la Universidad. Revista de Occidente S.A. Madrid. Alianza Editorial S.A. Madrid. 1999.

SAMPEDRO, J.L. (1997).- El salto a la tarima. El veterano. Gaceta Universitaria:19. 13 de enero de 1997.

TORTELLA, G. \& al. (2001).- Carta abierta a la ministra de educación sobre la L.R.U. El País, 9 de junio de 2001.

YELA, J.L. (2000).- Docencia universitaria e investigación: ¿complemento o esquizofrenia? Revista Vivat Academia http//www2.alcala.es/vivatacademia/diecinueve/opinion.htm 